Elsevier required licence: (c) $<2017>$. This manuscript version is made available under the CC-BY-NC-ND 4.0 license http://creativecommons.org/licenses/bync-nd/4.0/ 


\section{Title: Improving delirium recognition and assessment for people receiving inpatient palliative care: a mixed methods meta-synthesis}

\section{Authors}

Annmarie Hosie ${ }^{1}$

Meera Agar ${ }^{1}$

Elizabeth Lobb ${ }^{2}$

Patricia M Davidson ${ }^{3}$

Jane Phillips ${ }^{1}$

\section{Institutions}

1. IMPACCT - Improving Palliative, Aged and Chronic Care through Clinical

Research and Translation

University of Technology Sydney (UTS)

Faculty of Health

Building 10, Level 3

235-253 Jones $\mathrm{St}$

Ultimo, NSW 2007

Australia

2. Calvary Health Care Sydney

Palliative Care Department

91-111 Rocky Point Rd

Kogarah NSW 2217

Australia

3. Johns Hopkins University

School of Nursing

525 N. Wolfe Street

Baltimore MD 21205

United States

\section{Corresponding author}

Annmarie Hosie

Phone: +61 295144404

Email: annmarie.hosie@uts.edu.au

\section{Document details}

Number of text boxes: 1

Number of tables: 2

Number of figures: 1

Number of references: 75

Word count: 3189 
Delirium recognition and assessment in palliative care: a mixed methods study

\begin{abstract}
Background

Delirium is a serious acute neurocognitive condition frequently occurring for hospitalized patients, including those receiving care in specialist palliative care units. There are many delirium evidence-practice gaps in palliative care, including that the condition is under-recognized and challenging to assess.
\end{abstract}

\title{
Objectives
}

To report the meta-synthesis of a research project investigating delirium epidemiology, systems and nursing practice in palliative care units.

\section{Methods}

The Delirium in Palliative Care (DePAC) project was a two-phase sequential transformative mixed methods design with knowledge translation as the theoretical framework. The project answered five different research questions about delirium epidemiology, systems of care and nursing practice in palliative care units. Data integration and meta-synthesis occurred at project conclusion.

\section{Results}

There was a moderate to high rate of delirium occurrence in palliative care unit populations; and palliative care nurses had unmet delirium knowledge needs and worked within systems and team processes that were inadequate for delirium recognition and assessment. The meta-inference of the DePAC project was that a widely-held but paradoxical view that palliative care and dying patients are different from the wider hospital population has separated them from the overall generation of delirium evidence, and contributed to the extent of practice deficiencies in palliative care units.

\section{Conclusion}

Improving palliative care nurses' capabilities to recognize and assess delirium will require action at the patient and family, nurse, team and system levels. A broader, hospital-wide perspective would accelerate implementation of evidence-based delirium care for people receiving palliative care, both in specialist units, and the wider hospital setting. 
Delirium recognition and assessment in palliative care: a mixed methods study

\section{Keywords}

Assessment; Delirium; Inpatients; Palliative Care; Screening.

\section{Background}

Delirium is an acute neurocognitive condition of physiological origin frequently occurring in hospitalized patients (Text Box 1) (American Psychiatric Association, 2013). Patients of older age, with advanced or serious illness and/or cognitive impairment are at highest risk of delirium, leading to many adverse consequences: distress, increased complications (i.e. falls, further functional and cognitive decline), increased length of stay, mortality and healthcare costs (National Clinical Guideline Centre for Acute and Chronic Conditions, 2010). Families report distress and worry during episodes of delirium, and desire more timely information about what is happening and what to do (Day and Higgins, 2016, O'Malley et al., 2008). Clinicians also experience distress, uncertainty and are at times overly confident about what they consider is best delirium care (Agar et al., 2012, Brajtman et al., 2006).

\section{Implications of delirium in palliative care}

Delirium is also a source of suffering for people receiving palliative care in hospital, and their families. The sudden decline in awareness and cognition adversely impacts on capacity to make decisions, function and communicate, and exacerbates the fears and losses of advanced illness (O'Malley et al., 2008). Here, there can be up to six underlying causes of a delirium (Meagher et al., 2011). Causes include medical interventions, such as psychoactive medication to manage pain and nausea (Caraceni, 2013). A determined investigative approach is needed to optimize resolution of delirium, but assessment is challenging when the person is frail, fatigued, breathless or has difficulty communicating (Leonard et al., 2014). Clinical uncertainty about potential for its resolution in the last months, weeks and days of life means decision making about investigation and intervention is also not easy (Lawlor et al., 2000).

\section{Delirium evidence-practice gaps in palliative care}

The challenges, risks and suffering of delirium require palliative care clinicians to be highly skilled in recognizing and assessing its presence to ensure care that aligns with the person's needs and preferences (World Health Organisation, 2002). Yet delirium 
Delirium recognition and assessment in palliative care: a mixed methods study

\section{Text Box 1: DSM-5 diagnostic criteria for delirium}

A. Disturbed attention (i.e. reduced ability to focus, sustain or shift attention) and awareness (reduced orientation to the environment)

B. Disturbance developed over a short period of time (usually hours to a few days), represents a change from baseline attention and awareness, and tends to fluctuate in severity during the course of the day

C. An additional disturbance in cognition e.g. memory deficit, disorientation, language, visuospatial ability, or perception

D. The disturbances in Criteria $\mathrm{A}$ and $\mathrm{C}$ are not better explained by another preexisting, established, or evolving neurocognitive disorder and do not occur in the context of a severely reduced level of arousal, such as coma

E. Evidence from the history, physical examination, or laboratory findings that the disturbance is a direct physiological consequence of another medical condition, substance intoxication or withdrawal (i.e. due to a drug of abuse or to a medication), or exposure to a toxin, or is due to multiple etiologies (American Psychiatric Association, 2013). 
Delirium recognition and assessment in palliative care: a mixed methods study

is poorly recognized by palliative care teams, especially the hypoactive subtype, which easily can be mistaken for depression or fatigue (Fang et al., 2008, Spiller and Keen, 2006). Under-recognition can be attributed in part to a lack of routine screening in this setting (Barnes et al., 2010, Irwin et al., 2008). Overall, the delirium knowledge base is limited in palliative care (Lawlor et al., 2014). Nonpharmacological strategies to prevent and treat delirium are not definitively established (Gagnon et al., 2012); while anti-psychotics and benzodiazepines have become mainstay pharmacological treatment, with little evidence of effectiveness and safety, and a huge variation in prescribing (Agar et al., 2008). Delirium incidence, duration and/or severity actually appear to worsen when people receiving palliative care are given anti-psychotics or sedative medication (Agar et al., 2017, Beller et al., 2015).

\section{Rationale for the DePAC project}

The Delirium in Palliative Care Project ('DePAC project') aimed to better understand the problem of delirium, its under-recognition and the challenges of assessment in specialist palliative care inpatient units ('palliative care units'). The doctoral research focused, in part, on nursing practice because of the extensive literature on nurses' poor recognition of delirium (McCarthy, 2003, Mistarz et al., 2011, Steis and Fick, 2008). Investigation of delirium epidemiology was also undertaken to confirm the extent of its occurrence in this setting; and of systems of care, because interdisciplinary strategies and organizational supports can improve delirium practice and outcomes (Adams et al., 2015, Australian Commission on Safety and Quality in Health Care, 2013, Hshieh et al., 2015, Milisen et al., 2005, Naughton et al., 2005, Siddiqi et al., 2016).

The project began with the premise that evidence was required in palliative care inpatient unit systems to improve nurses' delirium recognition and assessment.

The complete DePAC project is published elsewhere as a doctoral thesis (Hosie, 2015). This paper presents the meta-synthesis and key finding of the overall project, which informs how a new perspective will accelerate translation of delirium evidence in palliative care. 
Delirium recognition and assessment in palliative care: a mixed methods study

\section{Aim}

To report the meta-synthesis of a research project investigating delirium epidemiology, systems and nursing practice in palliative care units.

\section{Methods}

\section{Design and theoretical framework}

The DePAC project was a two-phase sequential transformative mixed methods design (Creswell, 2009). Mixed methods were used because the project's five research questions were best answered by using both quantitative (QUANT) and qualitative (QUAL) methods, i.e. for complementarity (Halcomb and Andrew, 2009); and because complex problems are more fully understood by integrating multiple data (Creswell, 2009). Sequential denotes discrete phases: Phase one investigated delirium epidemiology and systems: QUANT + QUAL + QUANT; Phase two explored nursing practice: QUAL + QUAL. Transformative denotes that the research used a theoretical framework to address an issue for a marginalized or underrepresented population, with the intent to make an informed call for change (Creswell, 2009). Given the evidence-practice gaps, the theoretical framework chosen was knowledge translation (Graham et al., 2006).

\section{Samples, data collection and analysis}

The research was conducted in Australia during 2011-15. Participants were patients, nurses, physicians and allied health clinicians, educators and managers ('clinicians') located at palliative care units. The first author $[\mathrm{AH}]$ undertook data collection and all authors contributed to study planning and analysis.

\section{Studies}

Phase 1 studies were: a systematic review of delirium prevalence and incidence (Hosie et al., 2013); an environmental scan examining unit uptake of delirium guideline recommendations for recognition and assessment; and a cross-sectional study of delirium point-prevalence (Hosie et al., 2016). Phase 2 studies were: semistructured interviews to explore nurses' delirium recognition and assessment 
Delirium recognition and assessment in palliative care: a mixed methods study

experience, perceptions and capabilities, and barriers and enablers to optimal practice (Hosie et al., 2014, Hosie et al., 2014); and focus groups to obtain nurses' perceptions about using a brief screening tool, the Nursing Delirium Screening Scale (NuDESC) (Gaudreau et al., 2005, Hosie et al., 2015). (Table 1)

\section{Ethical considerations}

Ethical approvals were obtained from the St Vincent's Hospital Human Research Ethics Committee: reference numbers HREC/13/SVH/152 and LNR/12/SVH/336; and cross-institutional ratification from the University of Notre Dame Australia: reference number 013111S.

Approval for waiver of written patient consent for delirium screening and assessment was obtained for Study 3 (Adamis et al., 2005, Agar et al., 2013). Recruitment and consent of clinicians were undertaken by $\mathrm{AH}$ who had no managerial or existing collegial relationship with participants. Participant and site confidentiality and privacy were maintained through assignation of codes; storing signed consent forms and participant logs separately from other study data; and removal of names in transcripts.

\section{Positioning of the researchers}

The first author and doctoral researcher is a female registered nurse with long-term clinical experience across acute, community, palliative, and residential aged care settings, whose stance on palliative care aligns with the World Health Organization definition (2002). The supervision team are experienced researchers with nursing [JP and PD], medical [MA] and allied health [EL] expertise in palliative, aged and chronic care.

\section{Data analysis}

Analysis methods of the individual studies varied according to design (Hosie, 2015). This section describes mixed methods data analysis, which was a three-step process of interpretation and integration at project conclusion (Fetters et al., 2013), as follows:

1) Each research question was answered by results and findings of relevant studies. 
Delirium recognition and assessment in palliative care: a mixed methods study

2) Study results and findings were distilled, whereby the key quantitative (concerning epidemiological) and qualitative (concerning systems and practice) data were determined.

3) Meta-synthesis was undertaken, guided by Erzberger \& Kelle's complementarity model of triangulation (2003). Meta-synthesis is analogous to triangulation: "the combinations and comparisons of multiple data sources, data collection and analysis procedures, research methods, and/or inferences" (Tashakkori and Teddlie, 2003 p. 717). Results and findings were assigned equal weight, and compared and contrasted to determine whether there was correspondence or contradiction. The resulting determination was interpreted alongside the beginning inference, and the overall conclusion, termed the 'meta-inference', was generated (Erzberger and Kelle, 2003, Ostlund et al., 2011). Meta-synthesis thereby integrated both empirical data and theoretical understanding, i.e. beginning inference + QUANT + QUAL = meta-inference.

\section{Results}

Answers to the five research questions are provided in the full project report (Hosie, 2015). Here, key data (Table 1) and findings of the meta-synthesis are reported.

\section{Distilling results and findings}

\section{Epidemiology of delirium in palliative care units (QUANT)}

Palliative care unit patients were primarily an older advanced cancer population at risk of delirium. There was moderate-high delirium occurrence which provides a strong argument for routine screening and assessment in palliative care units. However, evidence for the impact, acceptability and potential harms of screening, as well as effective delirium treatment, is also needed (Hosie et al., 2013, Hosie et al., 2016).

\section{Systems (QUAL)}

Systems were inadequately informed and structured, and did not support nurses to optimally recognize and assess delirium in this setting. 
Delirium recognition and assessment in palliative care: a mixed methods study

Most delirium guidelines excluded palliative care populations, evidence and/or recommendation, despite stating the association between delirium and mortality (Australian Commission on Safety and Quality in Health Care, 2014, Barr et al., 2013, Care of the Confused Hospitalised Older Persons Study, 2010, Clinical Epidemiology and Health Service Evaluation Unit Melbourne Health, 2006, Michaud et al., 2007, National Clinical Guideline Centre for Acute and Chronic Conditions, 2010, Royal College of Physicians and British Geriatrics Society, 2006). Of three palliative care delirium guidelines, two were consensus-based only (Department of Health and Human Services Tasmania, 2009, Palliative Care Expert Group, 2010); and the third made many consensus-based recommendations (Canadian Coalition for Seniors' Mental Health, 2010). Unit level systems to support recognition and assessment of delirium were either absent, or constructed by different disciplines without team connection; i.e. team functioning was multidisciplinary, not interdisciplinary. Patients and families were not at the center of delirium recognition and assessment practice, or routinely informed or included. Where there was semblance of a system, it was only sporadically performed. Participants acknowledged that wider organizational guidance and direction were necessary for practice change generally, but largely absent with respect to delirium care (Hosie et al., 2014, Hosie et al., 2014).

\section{Nursing practice (QUAL)}

Nurses were aware of patients' delirium symptoms, and felt concern, compassion, surprise, puzzlement, frustration, isolation and overburdened. They did not conceptualize or communicate delirium according to diagnostic criteria, and often used imprecise terms such as 'terminal agitation' or 'terminal restlessness', even when patients were not imminently dying. This terminology led to inadequate nursing assessment of delirious patients and delayed medical follow up. Relieving distress was a priority and frequently, intervention (including pharmacological) occurred before comprehensive assessment. Patient observation primarily occurred during care delivery, underscoring the potential utility of brief bedside delirium tools. Participants believed rapport, trust and communication with patients and families helped them to recognize and understand the meaning of cognitive changes. The role of the nurse in delirium recognition and assessment was not defined and neither were structured interdisciplinary team processes in place. Nurses often hesitated to communicate 
Delirium recognition and assessment in palliative care: a mixed methods study

delirium observations to physicians and nursing colleagues and at times had experienced a lack of respect or response when they did so. They were aware of their delirium knowledge needs and requested point-of care guidance and more opportunities for education relevant to palliative care (Hosie et al., 2014, Hosie et al., 2015, Hosie et al., 2014).

\section{Meta-synthesis}

The beginning inference of the DePAC project was that delirium evidence was required in systems of palliative care units to improve recognition and assessment by nurses. Congruent with this premise, quantitative data confirmed a moderate to high rate of delirium occurrence in palliative care inpatients. Qualitative data revealed nurses' unmet delirium knowledge needs and inadequate systems, guidance and interdisciplinary team processes for delirium recognition and assessment, and thereby also confirmed the need for integration of delirium evidence. Yet there was contradiction between the rate of delirium occurrence in palliative care units and the inadequate knowledge, systems, practice, and communication. It was also puzzling that delirium guidelines consistently viewed palliative care patients as being separate and outside of their scope, given the epidemiology of delirium in specialist units, the many patients with life-threatening illness cared for throughout hospitals (Currow et al., 2008, To et al., 2011), and the association between delirium and mortality.

The meta-inference of the DePAC project was that a widely-held but paradoxical view that palliative care and dying patients are different from the wider hospital population has separated them from the overall generation of delirium evidence, and contributed to the extent of practice deficiencies in palliative care units. Conceivably, delirium evidence-practice gaps extend hospital-wide for patients with lifethreatening illness and palliative care needs.

Figure 1 presents the relationships between the beginning and ending theoretical understandings and the DePAC project data. Solid lines represent correspondence and the broken lines, contradiction. 
Delirium recognition and assessment in palliative care: a mixed methods study

\section{Discussion}

The reasons, implications, and remedy for the separation of palliative care and dying patients from delirium knowledge are discussed.

\section{Separating palliative care}

Modern hospice and palliative care units emerged in the 1960's with the opening of St Christopher's Hospice in London (St Christopher's, 2017). Palliative care was motivated to be a specialty because of the need and desire to improve control of distressing cancer-related symptoms, help people live until they die, and provide more holistic care (Twycross, 2016). In many respects, these goals are being realized with improvements in treatment and pain and symptom management. However, an unintended consequence of establishing discrete specialist units has been that palliative care is often viewed within healthcare, and the wider community, as confined to these specialist settings and people with cancer. In reality, patients with palliative care needs are located throughout hospital and community settings and have various life-threatening illnesses and other comorbidities (Worldwide Palliative Care Alliance, 2014).

Delirium has also been conceptualized differently according to where it occurs in the hospital setting. Guideline developers have adopted the separatist paradigm, and presumed that palliative care patients have different needs and that the specialty is best placed to determine what constitutes best delirium care for people with life threatening illness, who are dying and/or receiving palliative care.

\section{Palliative care practice with limited knowledge, language and systems}

Working alone, the specialty of palliative care has not determined what is best delirium care during life-threatening illness and dying (Lawlor et al., 2014). One reason there remains so many unanswered questions about delirium epidemiology, prevention, identification, management and supportive care in the specialty palliative care setting is that these patients represent only a small proportion of the overall hospital population; for example, in Australia they represent only $0.6 \%$ (Australian Institute of Health and Welfare, 2014). Another reason is that it is ethically and practically challenging to conduct research with people who are frail, delirious and near to dying. Yet recent research demonstrates it is both possible and necessary to 
Delirium recognition and assessment in palliative care: a mixed methods study

include these people in appropriate studies (Adamis et al., 2005, Agar et al., 2017, Currow, 2010, Holt et al., 2008).

Evidence-practice gaps were also expressed by use of a distinct local language for delirium. The terms 'terminal agitation' and 'terminal restlessness' were often used and revealed that clinicians conceptually linked delirium with dying (Brajtman, 2005, Heyse-Moore, 2003, Hosie et al., 2014). Elsewhere, specialization of care has resulted in many imprecise terms for delirium. For example, 'ICU psychosis' (now discouraged in the critical care literature) (Girard et al., 2008); 'sundowning'; and the ubiquitous 'confusion' (Morandi et al., 2009). None capture the full features of delirium; all downplay its significance and severity and contribute to its underrecognition. Shared understanding and better communication of delirium across teams, disciplines and settings will be achieved by using its correct name and scientifically precise criteria, such as contained in the American Psychiatric Association Diagnostic and Statistical Manual of Mental Disorders, Fifth Edition (APA-DSM-5) (Text Box 1). Unfortunately, the APA-DSM-5 manual is expensive and not easily accessible for clinicians.

Lacking an evidence base for delirium management, palliative care teams have responded to its frequency and their desire to relieve the suffering and disruption it entails by developing local ways of knowing and acting. These may be to the detriment of patients. For example, an ethnographic study of delirium practice in a Canadian hospice unit (Wright et al., 2015) reported that clinicians encouraged family members of dying patients with distressing delirious behaviors to think that they were no longer seeing the person they knew and loved, and even that they were to some extent "already dead" (p. 963). Clinicians believed that a helpful and compassionate strategy was to explain to a family that becoming “...withdrawn, confused, somnolent, or restless..." (p.962) was normal for a hospice patient (Wright et al., 2015). While these statements were intended to relieve a family's distress, they may also have had unintended and potentially harmful consequences. Firstly, conceptualizing delirium as normal during dying, rather than as common, abnormal and potentially reversible, may deny the patient and family the time and opportunity to address what is especially important to them (Steinhauser et al., 2000). Secondly, nihilistic approaches can be self-fulfilling. Most critically, seeing patients as being to 
Delirium recognition and assessment in palliative care: a mixed methods study

some extent already dead runs counter to the goals of palliative care, which include caring for and helping the person to live as actively as possible until death.

Another evidence-practice gap revealed by our research was that comprehensive assessment of delirious patients rarely was undertaken prior to administering antipsychotics and sedative medication (Hosie et al., 2014). This practice is problematic because pharmacological intervention as a response to 'terminal agitation' or 'terminal restlessness' is not rational prescribing based on science, for these terms do not describe a discrete medical condition. There are many possible causes for patient agitation and distress, including pain, urinary retention, anguish, fear, metabolic disturbance and/or drug intoxication, each requiring different means of relief. Secondly, neither antipsychotics nor sedatives are proven effective in preventing delirium or treating it once it occurs, and may even increase the severity of its symptoms (Agar et al., 2017, Lonergan et al., 2009, Neufeld et al., 2016, Siddiqi et al., 2016). Further challenging the long-standing palliative care practice of pharmacological intervention for delirium, a recent Cochrane review of 14 studies of palliative sedation reported insufficient evidence of its efficacy to improve symptom control or quality of life, including with respect to delirium (Beller et al., 2015). These results highlight the need for alternative ways to relieve distress during delirium.

\section{Including patient, family, team and external perspectives in local action}

Much of current palliative care unit delirium practice is at odds with what people most value at the end of life, which is to be mentally aware, safe, and maintain a sense of self (Collier et al., 2016, Spichiger, 2008, Steinhauser et al., 2000). Families of delirious patients feel best supported when they are consulted and given timely information, the patient is seen as a person and their needs are met, and respect and understanding is shown for their subjective experience of delirium (Bolton et al., 2016, O'Malley et al., 2008). The DePAC project found that patients and families were not included in delirium recognition and assessment nor routinely informed, a finding which is common across settings of care (O'Malley et al., 2008). More promisingly, Bolton et al (2016) reported a recent quality improvement initiative in a New Zealand hospice to improve care for people with cognitive impairment. The "Te Kete Marie" was developed by an interdisciplinary team with community partners, 
Delirium recognition and assessment in palliative care: a mixed methods study

and included a cognitive assessment tool, team education, patient and family activities and reality orientation equipment. Family carers perceived that staff responded to the patient as an individual, included family, promoted comfort, safety, flexibility, privacy and access to outdoors; and where possible, prepared for discharge (Bolton et al., 2016). This exemplar local quality initiative was centered on the needs of patients and families, informed by evidence and external partners and, importantly, reported feedback from family about its impact (Bolton et al., 2016).

Improving delirium care at the end of life therefore requires a transformative agenda in palliative care, particularly on the meaning, etiology, and significance of the experience. The call for change of the DePAC project is that people receiving palliative care be included in future delirium research and the scope of hospital-wide guidelines. Also, that palliative care units adapt, test and implement wider delirium knowledge. Incorporating evidence-based delirium organizational initiatives, such as the new Australian delirium clinical care standard, could provide much needed frameworks for the fundamentals of delirium care, including screening and assessment processes (Australian Commission on Quality and Safety of Healthcare, 2017).

\section{Strengths and Limitations}

With the exception of the systematic review, data were derived primarily from the Australian impatient palliative care setting. Findings may not be transferable to other settings of care, including palliative care inpatient services that are situated within other cultures, healthcare systems or geographical locations. The focus was on delirium recognition and assessment, not the full clinical care pathway which must include prevention and treatment.

The research was descriptive and the meta-inference is hypothesis generating only. The 'how to' of mixed methods data integration is not fully established, meaning it is more challenging to defend the rigor of the meta-synthesis. Use of a model of triangulation appropriate to the mixed methods design, along with the researchers' understanding of the context and culture of palliative care, supports the logic and trustworthiness of the findings (Fetters et al., 2013). 
Delirium recognition and assessment in palliative care: a mixed methods study

\section{Conclusion}

Improving specialist inpatient palliative care nurses' capabilities to recognize and assess delirium will require action at the patient and family, nurse, team and system levels. A broader, hospital-wide perspective would accelerate implementation of evidence-based delirium care for people receiving palliative care, both in specialist units, and the wider hospital setting.

\section{Acknowledgements}

Dr Hosie was supported in her doctoral research at the University of Notre Dame Australia though an Australian Postgraduate Award from the Commonwealth Government of Australia (2012-2015).

The authors gratefully acknowledge the patients, clinicians and managers who contributed to and supported this research. 
Table 1: The DePAC project research questions, phases and studies

\begin{tabular}{|c|c|}
\hline \multirow[t]{2}{*}{ Research questions } & $\begin{array}{l}\text { 1. What is the epidemiology of delirium in the palliative care unit population? } \\
\text { 2. Is delirium recognition and assessment guidance available to nurses working in palliative care units? } \\
\text { 3. What are the experiences, views and capabilities of nurses in recognising and assessing palliative care } \\
\text { inpatients' delirium? } \\
\text { 4. What are the barriers and enablers to nurses recognising and assessing delirium in palliative care units? } \\
\text { 5. What is required to improve the capabilities of nurses to recognise and assess delirium in palliative care } \\
\text { units? }\end{array}$ \\
\hline & Phase 1 (QUANT + QUAL + QUANT) \\
\hline \multirow[t]{4}{*}{ Studies } & $\begin{array}{l}\text { 1. Systematic review of delirium epidemiology studies in palliative care unit populations. } \\
\text { 2. Environmental scan to investigate palliative care unit uptake of guideline recommendations for delirium } \\
\text { recognition and assessment, involving i) a snowball search for delirium guidelines and ii) group } \\
\text { interviews with purposive sample of physicians, nurses, allied health professionals, educators and } \\
\text { managers 'key informants' of three palliative care units. } \\
\text { 3. Prospective cross sectional study to estimate } 24 \text {-hour delirium point-prevalence in an Australian } \\
\text { palliative care unit population, and test feasibility and acceptability of the delirium measures. }\end{array}$ \\
\hline & Phase 2 (QUAL + QUAL) \\
\hline & $\begin{array}{l}\text { 4. Semi-structured interviews using critical incident technique to identify i) nurses' delirium recognition } \\
\text { and assessment experiences, perceptions, and capabilities; and ii) facilitators and enablers to delirium } \\
\text { recognition and assessment in palliative care units. } \\
\text { 5. Focus groups to identify nurses' perceptions of the feasibility of the Nursing Delirium Screening Scale } \\
\text { in palliative care unit practice. }\end{array}$ \\
\hline & Mixed methods data integration and meta-synthesis \\
\hline
\end{tabular}


Table 2: Key results and findings

\begin{tabular}{|c|c|c|}
\hline Epidemiology: QUANT & Systems: QUAL & Nursing practice: QUAL \\
\hline Studies 1 and 3 & Studies 2,4 and 5 & Studies 4 and 5 \\
\hline $\begin{array}{l}\text { - Older, mostly advanced cancer } \\
\text { population, at risk of delirium } \\
\text { - Prevalence: } 13-42 \% \text { at admission; } 26- \\
62 \% \text { during admission; } 59-88 \% \text { in weeks } \\
\text { or hours before death } \\
\text { - Incidence: } 3-45 \% \\
\quad \text { when screened at least daily: } 33- \\
45 \% \\
\text { - Hypoactive subtype most common: } 68 \text { - } \\
86 \% \text { of all cases }\end{array}$ & $\begin{array}{l}\text { Delirium guidelines mostly excluded or } \\
\text { omitted reference to palliative care } \\
\text { patients, evidence and/or end-of-life care; } \\
\text { and palliative care delirium guidelines } \\
\text { were largely consensus based } \\
\text { - No organizational direction for delirium } \\
\text { care } \\
\text { - No routine processes for delirium } \\
\text { recognition and assessment } \\
\text { - Multidisciplinary practice and learning, } \\
\text { not interdisciplinary } \\
\text { - Patients and families were not at the } \\
\text { center of delirium recognition and } \\
\text { assessment practice nor routinely } \\
\text { informed or included. } \\
\text { - Scope to build, adapt and integrate } \\
\text { delirium knowledge into palliative care } \\
\text { unit systems }\end{array}$ & $\begin{array}{l}\text { - Delirious patients elicited feelings of } \\
\text { concern, compassion, puzzlement, } \\
\text { frustration, uncertainty, isolation and } \\
\text { burden for nurse participants } \\
\text { - Changes signifying delirium were } \\
\text { recognized but not framed according to } \\
\text { diagnostic criteria } \\
\text { - Imprecise language, e.g. 'terminal } \\
\text { agitation', 'terminal restlessness' } \\
\text { - Sought to rapidly relieve distress, often } \\
\text { without comprehensive assessment } \\
\text { - Brief, simple tools, point-of-care } \\
\text { guidance and interdisciplinary education } \\
\text { needed and requested } \\
\text { - Scope to develop nurses' communication, } \\
\text { collaboration, role and leadership for } \\
\text { delirium care within the interdisciplinary } \\
\text { team }\end{array}$ \\
\hline
\end{tabular}


Delirium recognition and assessment in palliative care: a mixed methods study

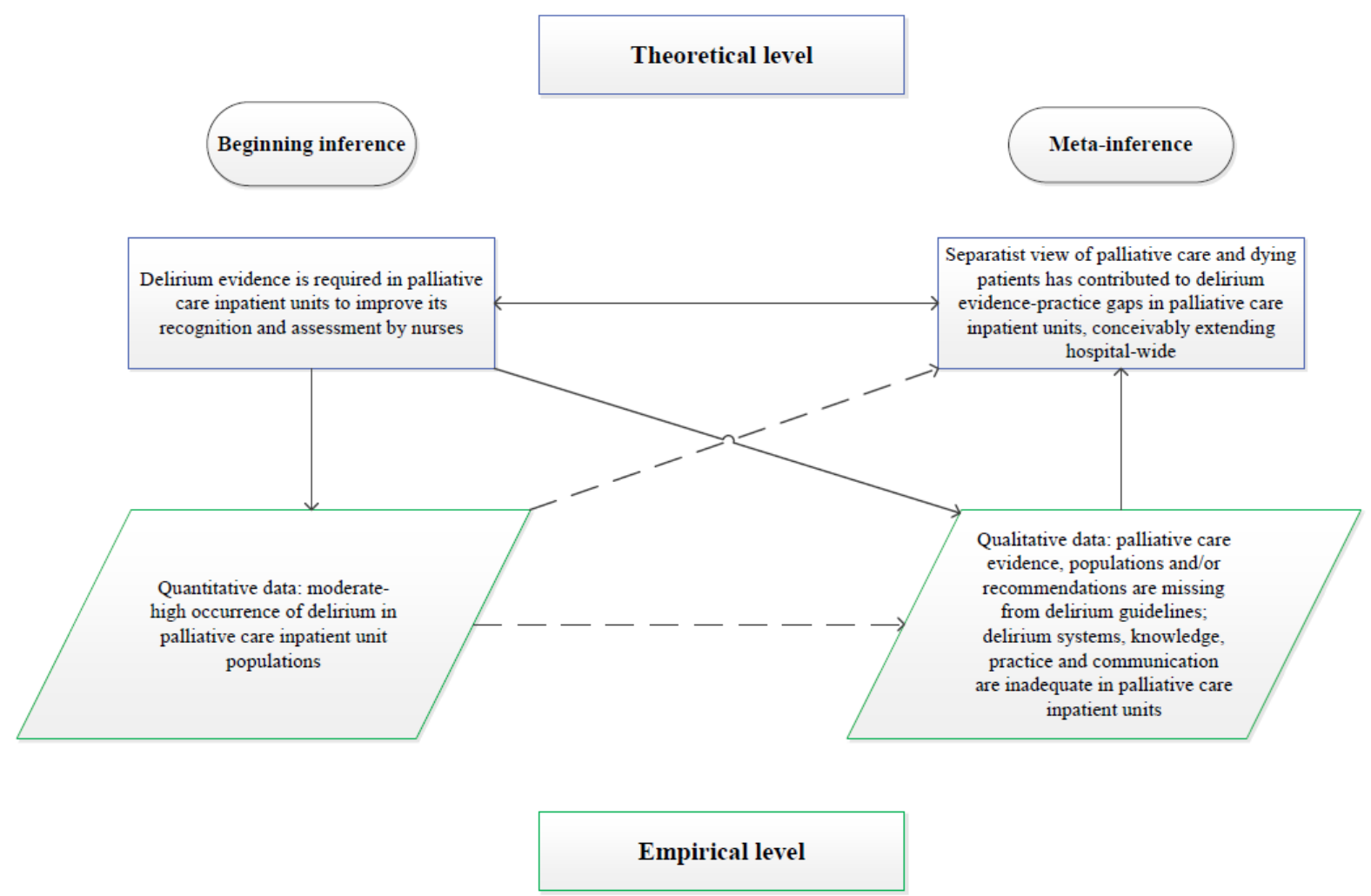

Figure 1: Meta-synthesis of the DePAC project 
Delirium recognition and assessment in palliative care: a mixed methods study

\section{References}

Adamis, D., Martin, F.C., Treloar, A., Macdonald, A.J.D., 2005. Capacity, consent, and selection bias in a study of delirium. Journal of Medical Ethics 31 (3), 137-143.

Adams, C.L., Scruth, E.A., Andrade, C., Maynard, S., Snow, K., Olson, T.L., Ingerson, S.D., Duffy, B.A., Cheng, E., 2015. Implementing clinical practice guidelines for screening and detection of delirium in a 21-hospital system in northern California: real challenges in performance improvement. Clinical Nurse Specialist 29 (1), 29-37.

Agar, M., Currow, D.C., Plummer, J., Chye, R., Draper, B., 2008. Differing management of people with advanced cancer and delirium by four subspecialties. Palliative Medicine 22 (5), 633-640.

Agar, M., Draper, B., Phillips, P.A., Phillips, J., Collier, A., Harlum, J., Currow, D., 2012. Making decisions about delirium: A qualitative comparison of decision making between nurses working in palliative care, aged care, aged care psychiatry, and oncology. Palliative Medicine 26 (7), 887-896.

Agar, M., Ko, D.N., Sheehan, C., Chapman, M., Currow, D.C., 2013. Informed consent in palliative care clinical trials: challenging but possible. Journal of Palliative Medicine 16 (5), 485-491.

Agar, M., Lawlor, P.G., Quinn, S., et al., 2017. Efficacy of oral risperidone, haloperidol, or placebo for symptoms of delirium among patients in palliative care: A randomized clinical trial. JAMA Internal Medicine 177 (1), 34-42.

American Psychiatric Association, 2013. Diagnostic and Statistical Manual of Mental Disorders, Fifth Edition (DSM-5). American Psychiatric Publisher, Arlington, VA.

Australian Commission on Quality and Safety of Healthcare, 2017. Delirium Clinical Care Standard. Sydney.

Australian Commission on Safety and Quality in Health Care, 2014. A better way to care: Safe and high-quality care for patients with cognitive impairment (dementia and delirium) in hospital - Actions for clinicians. ACSQHC, Sydney.

Australian Commission on Safety and Quality in Health Care, 2013. Evidence for the safety and quality issues associated with the care of patients with cognitive impairment in acute care settings: a rapid review. ACSQHC, Sydney.

Australian Institute of Health and Welfare, 2014. Palliative care services in Australia 2014. AIHW, Canberra.

Barnes, J., Kite, S., Kumar, M., 2010. The recognition and documentation of delirium in hospital palliative care inpatients. Palliative and Supportive Care 8 (2), 133136.

Barr, J., Fraser, G.L., Puntillo, K., Ely, E.W., Gélinas, C., Dasta, J.F., Davidson, J.E., Devlin, J.W., Kress, J.P., Joffe, A.M., Coursin, D.B., Herr, D.L., Tung, A., Robinson, B.R.H., Fontaine, D.K., Ramsay, M.A., Riker, R.R., Sessler, C.N., Pun, B., Skrobik, Y., Jaeschke, R., 2013. Clinical practice guidelines for the management of pain, agitation, and delirium in adult patients in the intensive care unit. Critical Care Medicine 41 (1), 263-306.

Beller, E.M., van Driel, M.L., McGregor, L., Truong, S., Mitchell, G., 2015. Palliative pharmacological sedation for terminally ill adults. Cochrane Database of Systematic Reviews (1). 
Delirium recognition and assessment in palliative care: a mixed methods study

Bolton, L., Loveard, T., 2016. Te Kete Marie - the peaceful basket: an initiative for supporting people with dementia or delirium. International Journal of Palliative Nursing 22 (3), 130-136.

Bolton, L., Loveard, T., Brander, P., 2016. Carer experiences of inpatient hospice care for people with dementia, delirium and related cognitive impairment. International Journal of Palliative Nursing 22 (8), 396-403.

Brajtman, S., 2005. Terminal restlessness: perspectives of an interdisciplinary palliative care team. International Journal of Palliative Nursing 11 (4), 170, 172-178.

Brajtman, S., Higuchi, K., McPherson, C., 2006. Caring for patients with terminal delirium: palliative care unit and home care nurses' experiences. International Journal of Palliative Nursing 12 (4), 150-156.

Canadian Coalition for Seniors' Mental Health, 2010. Guideline on the Assessment and Treatment of Delirium in Older Adults at the End of Life. Canadian Coalition for Seniors' Mental Health (CCSMH), Toronto.

Caraceni, A., 2013. Drug-associated delirium in cancer patients. European Journal of Cancer Supplements 11 (2), 233-240.

Care of the Confused Hospitalised Older Persons Study, 2010. Delirium. NSW Government.

Clinical Epidemiology and Health Service Evaluation Unit Melbourne Health, 2006. Clinical Practice Guidelines for the Management of Delirium in Older People. Australian Health Ministers' Advisory Council, Melbourne.

Collier, A., Sorensen, R., Iedema, R., 2016. Patients' and families' perspectives of patient safety at the end of life: a video-reflexive ethnography study. International Journal for Quality in Health Care 28 (1), 66-73.

Creswell, J.W., 2009. Research Design: Qualitative, quantitative, and mixed methods approaches. Sage Publications, Inc., Thousand Oaks.

Currow, D.C., 2010. Why don't we do more rigorous clinical research so that we can stop experimenting on patients? Journal of Palliative Medicine 13 (6), 636637.

Currow, D.C., Burns, C.M., Abernethy, A.P., 2008. Place of death for people with noncancer and cancer illness in South Australia: A population-based survey. Journal of Palliative Care 24 (3), 144-150.

Day, J., Higgins, I., 2016. Mum's absence(s): conceptual insights into absence as loss during a loved one's delirium. Journal of Clinical Nursing 25 (13-14), 20662073.

Department of Health and Human Services Tasmania, 2009. Palliative Care Clinical Management Guidelines Delirium.

Erzberger, C., Kelle, U., 2003. Making inferences in mixed methods: The rules of integration. In: Tashakkori, A., Teddlie, C. (Eds.), Handbook of Mixed Methods in Social and Behavioural Research. Sage Publications, Inc, Thousand Oaks, pp. 457-488.

Fang, C.K., Chen, H.W., Liu, S.I., Lin, C.J., Tsai, L.Y., Lai, Y.L., 2008. Prevalence, detection and treatment of delirium in terminal cancer inpatients: A prospective survey. Japanese Journal of Clinical Oncology 38 (1), 56-63.

Fetters, M.D., Curry, L.A., Creswell, J.W., 2013. Achieving Integration in Mixed Methods Designs-Principles and Practices. Health Services Research 48 (6pt2), 2134-2156. 
Gagnon, P., Allard, P., Gagnon, B., Mérette, C., Tardif, F., 2012. Delirium prevention in terminal cancer: assessment of a multicomponent intervention. PsychoOncology 21 (2), 187-194.

Gaudreau, J.D., Gagnon, P., Harel, F., Tremblay, A., Roy, M.A., 2005. Fast, systematic, and continuous delirium assessment in hospitalized patients: the nursing delirium screening scale. Journal of Pain and Symptom Management 29 (4), 368-375.

Girard, T.D., Pandharipande, P.P., Ely, E.W., 2008. Delirium in the intensive care unit. Critical Care 12 (Suppl 3), S3-S3.

Graham, I.D., Logan, J., Harrison, M.B., Straus, S.E., Tetroe, J., Caswell, W., Robinson, N., 2006. Lost in Knowledge Translation: Time for a Map? The Journal of Continuing Education in the Health Professions 26, 13-24.

Halcomb, E.J., Andrew, S., 2009. Managing Mixed Methods Projects. In: Andrew, S., Halcomb, E.J. (Eds.), Mixed Methods Research for Nursing and the Health Sciences. Wiley-Blackwell, Chichester.

Heyse-Moore, L., 2003. Terminal restlessness and sedation: A note of caution [4]. Palliative Medicine 17 (5), 469.

Holt, R., Siddiqi, N., Young, J., 2008. The ethics of consent in delirium studies. Journal of Psychosomatic Research 65 (3), 283-287.

Hosie, A., 2015. Delirium epidemiology, systems and nursing practice in palliative care inpatient settings: A descriptive mixed methods project (The DePAC Project) In: College of Nursing. University of Notre Dame Australia.

Hosie, A., Agar, M., Lobb, E., Davidson, P.M., Phillips, J., 2014. Palliative care nurses' recognition and assessment of patients with delirium symptoms: A qualitative study using critical incident technique. International Journal of Nursing Studies 51 (10), 1353-1365.

Hosie, A., Davidson, P.M., Agar, M., Sanderson, C.R., Phillips, J., 2013. Delirium prevalence, incidence, and implications for screening in specialist palliative care inpatient settings: A systematic review. Palliative Medicine 27 (6), 486498.

Hosie, A., Lobb, E., Agar, M., Davidson, P.M., Chye, R., Lam, L., Phillips, J., 2016. Measuring delirium point-prevalence in two Australian palliative care inpatient units. International Journal of Palliative Nursing 22 (1), 13-21.

Hosie, A., Lobb, E., Agar, M., Davidson, P.M., Chye, R., Phillips, J., 2015. Nurse perceptions of the Nursing Delirium Screening Scale in two palliative care inpatient units: a focus group study. Journal of Clinical Nursing, n/a-n/a.

Hosie, A., Lobb, E., Agar, M., Davidson, P.M., Phillips, J., 2014. Identifying the barriers and enablers to palliative care nurses' recognition and assessment of delirium symptoms: a qualitative study. Journal of Pain and Symptom Management 48 (5), 815-830.

Hshieh, T.T., Yue, J., Oh, E., Puelle, M., Dowal, S., Travison, T., Inouye, S.K., 2015. Effectiveness of multicomponent nonpharmacological delirium interventions: a meta-analysis. JAMA Internal Medicine 175 (4), 512-520.

Irwin, S.A., Rao, S., Bower, K.A., Palica, J., Rao, S.S., Maglione, J.E., Soskins, M., Betterton, A.E., Ferris, F.D., 2008. Psychiatric issues in palliative care: Recognition of delirium in patients enrolled in hospice care. Palliative and Supportive Care 6 (2), 159-164.

Lawlor, P.G., Davis, D.H.J., Ansari, M., Hosie, A., Kanji, S., Momoli, F., Bush, S.H., Watanabe, S., Currow, D.C., Gagnon, B., Agar, M., Bruera, E., Meagher, D., de Rooij, S.E.J.A., Adamis, D., Caraceni, A., Marchington, K.L., Stewart, 
Delirium recognition and assessment in palliative care: a mixed methods study

D.J., 2014. An Analytic Framework for Delirium Research in Palliative Care Settings: Integrated Epidemiological, Clinician-Researcher and Knowledge User Perspectives. Journal of Pain and Symptom Management 48 (2), 159175.

Lawlor, P.G., Gagnon, B., Mancini, I.L., Pereira, J.L., Hanson, J., Suarez-Almazor, M.E., Bruera, E.D., 2000. Occurrence, causes, and outcome of delirium in patients with advanced cancer: a prospective study. Archives of Internal Medicine 160 (6), 786-794.

Leonard, M., Nekolaichuk, C., Meagher, D.J., Barnes, C., Gaudreau, J.D., Watanabe, S., Agar, M., Bush, S.H., Lawlor, P.G., 2014. Practical Assessment of Delirium in Palliative Care. Journal of Pain and Symptom Management 48 (2), 176-190.

Lonergan, E., Luxenberg, J., Areosa Sastre, A., 2009. Benzodiazepines for delirium. In: Cochrane Database of Systematic Reviews. John Wiley \& Sons, Ltd, Chichester, UK.

McCarthy, M.C., 2003. Detecting acute confusion in older adults: Comparing clinical reasoning of nurses working in acute, long-term, and community health care environments. Research in Nursing and Health 26 (3), 203-212.

Meagher, D.J., Leonard, M., Donnelly, S., Conroy, M., Adamis, D., Trzepacz, P.T., 2011. A longitudinal study of motor subtypes in delirium: Relationship with other phenomenology, etiology, medication exposure and prognosis. Journal of Psychosomatic Research 71 (6), 395-403.

Michaud, L., Bula, C., Berney, A., Camus, V., Voellinger, R., Stiefel, F., Burnand, B., 2007. Delirium: Guidelines for general hospitals. Journal of Psychosomatic Research 62 (3), 371-383.

Milisen, K., Lemiengre, J., Braes, T., Foreman, M.D., 2005. Multicomponent intervention strategies for managing delirium in hospitalized older people: Systematic review. Journal of Advanced Nursing 52 (1), 79-90.

Mistarz, R., Eliott, S., Whitfield, A., Ernest, D., 2011. Bedside nurse-patient interactions do not reliably detect delirium: An observational study. Australian Critical Care 24 (2), 126-132.

Morandi, A., Solberg, L.M., Habermann, R., Cleeton, P., Peterson, E., Ely, E.W., Schnelle, J., 2009. Documentation and management of words associated with delirium among elderly patients in postacute care: a pilot investigation. Journal of the American Medical Directors Association 10 (5), 330-334.

National Clinical Guideline Centre for Acute and Chronic Conditions, 2010. Delirium: diagnosis, prevention and management, NICE Clinical Guideline 103. National Institute for Health and Clinical Excellence, London.

Naughton, B.J., Saltzman, S., Ramadan, F., Chadha, N., Priore, R., Mylotte, J.M., 2005. A multifactorial intervention to reduce prevalence of delirium and shorten hospital length of stay. Journal of the American Geriatric Society 53 (1), 18-23.

Neufeld, K.J., Yue, J., Robinson, T.N., Inouye, S.K., Needham, D.M., 2016. Antipsychotic Medication for Prevention and Treatment of Delirium in Hospitalized Adults: A Systematic Review and Meta-Analysis. Journal of the American Geriatric Society 64 (4), 705-714.

O'Malley, G., Leonard, M., Meagher, D., O'Keeffe, S.T., 2008. The delirium experience: a review. Journal of Psychsomatic Research 65 (3), 223-228.

Ostlund, U., Kidd, L., Wengstrom, Y., Rowa-Dewar, N., 2011. Combining qualitative and quantitative research within mixed method research designs: a 
Delirium recognition and assessment in palliative care: a mixed methods study

methodological review. International Journal of Nursing Studies 48 (3), 369383.

Palliative Care Expert Group, 2010. Delirium, dementia and other disorders of brain function in patients receiving palliative care. In, Therapeutic guidelines Palliative care. Therapeutic Guidelines Ltd, North Melbourne, Vic, pp. 290294.

Royal College of Physicians and British Geriatrics Society, 2006. The prevention, diagnosis and management of delirium in older people: National guidelines. Royal College of Physicians, Salisbury, UK.

Siddiqi, N., Harrison, J.K., Clegg, A., Teale, E.A., Young, J., Taylor, J., Simpkins, S.A., 2016. Interventions for preventing delirium in hospitalised non-ICU patients. Cochrane Database of Systematic Reviews (Issue 3. Art. No.: CD005563. ).

Spichiger, E., 2008. Living with terminal illness: patient and family experiences of hospital end-of-life care. International Journal of Palliative Nursing 14 (5), 220-228.

Spiller, J.A., Keen, J.C., 2006. Hypoactive delirium: assessing the extent of the problem for inpatient specialist palliative care. Palliative Medicine 20 (1), 1723.

St Christopher's, 2017. Dame Cicely Saunders: Her life and work.

Steinhauser, K.E., Christakis, N.A., Clipp, E.C., Mcneilly, M., McIntyre, L., Tulsky, J.A., 2000. Factors considered important at the end of life by patients, family, physicians, and other care providers. JAMA 284.

Steis, M.R., Fick, D.M., 2008. Are nurses recognizing delirium? A systematic review. Journal of Gerontological Nursing 34 (9), 40-49.

Tashakkori, A., Teddlie, C., 2003. Handbook of Mixed Methods Research in Social and Behavioral Research. SAGE Publications, Thousand Oaks.

To, T.H.M., Greene, A.G., Agar, M., Currow, D.C., 2011. A point prevalence survey of hospital inpatients to define the proportion with palliation as the primary goal of care and the need for specialist palliative care. Internal Medicine Journal 41 (5), 430-433.

Twycross, R., 2016. A tribute to Dame Cicely: St Christopher's.

World Health Organisation, 2002. WHO Definition of Palliative Care. World Health Organisation, pp. http://www.who.int/cancer/palliative/definition/en/.

Worldwide Palliative Care Alliance, 2014. Global Atlas of Palliative Care at the End of Life. World Health Organisation.

Wright, D.K., Brajtman, S., Cragg, B., Macdonald, M.E., 2015. Delirium as letting go: An ethnographic analysis of hospice care and family moral experience. Palliative Medicine 29 (10), 959-966. 